\title{
Introduzione / Introduction
}

Beatrice Barbalato

[...] nec corpus remanet, quondam quod amaverat Echo quae tamen ut vidit, quamvis irata memorque, indoluit, quotiensque puer miserabilis 'eheu' dixerat, haec resonis iterabat vocibus 'eheu'; cumque suos manibus percusserat ille lacertos, haec quoque reddebat sonitum plangoris eundem. ultima vox solitam fuit haec spectantis in undam: 'heu frustra dilecte puer!' totidemque remisit verba locus, dictoque vale 'vale' inquit et Echo

Ovidius, Metamorphoseon, Liber III, vv. 493-501 
L'ascoltare, il sentito dire, la phonè nei racconti di sé

Introduction $^{1}$

Le récit de soi, au-delà du mythe d'Echo - Le récit de soi: écouter, entendre, ouïr, 'oreiller'... a été le thème de la VIIIème rencontre de l'Osservatorio scientifico della memoria scritta, orale, filmica, e del patrimonio autobiografico, suivie de la session filmique Auto-biorégie. Cette initiative s'est déroulée au Castello Guevara (Bovino-Foggia) du 29 juillet au 5 août 2009.

Au cours de ces échanges entre disciplines différentes (histoire, sémiotique, linguistique, critique littéraire), celles-ci se croisent en un moment spécifique, l'idée maîtresse étant d'observer, d'analyser transversalement les formes de la communication autobiographique plutôt que ses contenus, la rhétorique du texte plutôt que la valeur de témoignage, en dépit naturellement du poids de ce dernier.

C'est le dialogue entre différentes expressions culturelles qui se trouve donc privilégié: la culture haute et $d u$ bas, écrite, orale, filmique, et théâtrale. L'italien, le français, l'espagnol, l'anglais sont les langues d'échange.

Je ferai état à présent de quelques traits et motifs qui ont mené au choix de ce thème pour le colloque 2009 ainsi que de réflexions sur les essaies publiés dans ce numéro de Mnemosyne $o$ la costruzione del senso.

Otobiographies - Nietzsche a consacré plusieurs exposés aux formes de la transmission du savoir institutionnel. En distinguant grande oreille et petite oreille, il a voulu marquer la distance entre l'oreiller/espionner versus écouter/entendre: «L'étudiant écoute [...] Une bouche qui parle, beaucoup d'oreilles et moitié moins de mains qui écrivent - voilà l'appareil académique extérieur, voilà la machine à culture de l'Université mise en activité.

Pour tout le reste le possesseur de cette bouche est séparé et indépendant des détenteurs des nombreuses oreilles $[\ldots] »$.

\footnotetext{
${ }^{1}$ Les références bibliographiques figurent aux pages recto dans la version italienne de ce texte.
} 
Introduzione/Introduction

Introduzione

Il racconto di sé, al di là del mito di Eco - L'ascoltare, il sentito dire, la phonè nei racconti di sé è stato il tema dell'VIII incontro dell'Osservatorio scientifico della memoria scritta, orale, filmica, e del patrimonio autobiografico, e della rassegna filmica Auto-bioregia ${ }^{2}$, tenuti a Bovino (Foggia) nel Castello Guevara dal 29 luglio al 5 agosto 2009. Durante questi colloqui internazionali annuali, che trattano ogni volta un tema specifico, si realizza un confronto fra studiosi provenienti da diverse discipline (storia, linguistica, semiotica, psicologia). L'idea guida è di osservare, di analizzare trasversalmente le forme di comunicazione autobiografica, piuttosto che i contenuti; la retorica del testo, piuttosto che il valore testimoniale, che certamente ha un suo rilievo.

Favorito in questi incontri è il dialogo fra cultura alta e dal basso, scritta, orale, filmica, e teatrale. L'italiano, il francese, l'inglese, e lo spagnolo sono le lingue d'uso ${ }^{3}$.

In ordine sparso ripercorro qualche sollecitazione che ha portato a scegliere questo tema per il colloquio 2009, e alcune riflessioni sui saggi che vengono pubblicati in questo numero.

Otobiografie - Nietzsche ha dedicato alcune conferenze alle forme di trasmissione del sapere istituzionale. La differenza fra grande orecchio e piccolo orecchio segna per Nietzsche il confine fra l'orecchiare/spiare versus ascoltare/intendere: «Lo studente ascolta [...] Una bocca che parla, molte orecchie e un numero di gran lunga minore di mani che scrivono ecco il dispositivo accademico esterno, ecco la macchina culturale dell'Università messa in moto.

Per tutto il resto il possessore di questa bocca è separato e indipendente dai detentori di diverse orecchie $[\ldots]{ }^{4}$.

${ }^{2}$ Questo appuntamento annuale è organizzato dall'ass. cult.:

Mediapolis-Europa (http://mediapoliseuropa.freehostia.com/), in collaborazione con diverse università europee e statunitensi, col sostegno del Comune di Bovino, della Regione Puglia, della Società cooperativa Sipario. Una selezione delle relazioni tenute durante il colloquio è pubblicata in questo numero 3 della rivista.

${ }^{3}$ Queste sono le lingue normalmente usate durante il colloquio, con delle traduzioni 'in successiva' laddove si rende necessario.

${ }^{4}$ Friedrich NIETZSCHE, «Cinquième conférence, - 23 Mars 1872», in Sur l'avenir de nos établissements d'enseignement, Testi e varianti a cura di G. COLLI e M. MONTINARI, tradotto dal tedesco da Jean-Louis BACKES, Michel HAAR e Marc B. DE LAUNAY, Paris, Gallimard, 1975, p. 152. (Adelphi, 1973). 
Jacques Derrida reprend ce thème dans Otobiographies, non auto, mais oto oreille: «Devenus tout ouïe pour ce chien de phonographe, vous vous transformez en poste récepteur à haute fidélité, et l'oreille, la vôtre qui est aussi celle de l'autre, se met à occuper dans votre corps la place disproportionnée de l' 'estropié à rebours'». On peut donc devenir un être estropié, réduit à ses seules oreilles. Le même thème parcourt Le Témoin auriculaire - Cinquante caractères d'Elias Canetti. Avec humour Canetti décrit le bruissement des commérages qui constituent la toile de fond des communautés fermées.

Un portrait d'enfance 'auriculaire' - Un récit, celui de Muriel - un texte de quarante pages, déposé aux Archives du Patrimoine Autobiographique de la Belgique -, donne un aperçu très clair de l'importance de l'ouïe dans les souvenirs d'enfance. L'arrivée de son père presque toujours ivre, violent envers sa femme, les enfants confinés dans leur chambre et entendant les cris -, était captée par ces gamins à travers la perception exacte de ses déplacements dans l'espace domestique: «Un soir quand mes sœurs et moi nous étions au lit, on entendait encore qu'il [le père] cassait tout dans la maison. Nous entendions ma mère crier et pleurer, puis on a entendu 'je vais te tuer'. Ma mère a commencé à courir dans la maison, puis plus rien. Nous entendions mon père crier au loin 'ne reviens plus', etc., ensuite il a couru dehors, et il a pris sa voiture».

De l'écoute à la parole comme signe - À plusieurs reprises durant le colloque, il a été rappelé que l'ouïr/l'entendre constituait une faculté primordiale, exactement selon le propos de Freud: «Le sens que l'enfant ne peut pas fermer». Un des sens pas toujours exploré, comme on le constate à travers l'analyse du nombre de lemmes qui le désignent. En latin l'écho est appelé imago vocis, en ramenant au visuel un phénomène auditif.

Le linguiste Tullio de Mauro a mis en évidence que les termes avec lesquels on désigne la voix (verba loquendi) sont relativement plus nombreux que les termes qui désignent l'ouïr et 
Jacques Derrida riprende questo tema in Otobiographies ${ }^{5}$, non auto, ma oto orecchio: «Divenuti tutto udito per questo cane di fonografo, voi vi trasformate in posto di ricezione ad alta fedeltà, e l'orecchio, il vostro che è anche quello dell'altro, comincia a occupare nel vostro corpo il posto sproporzionato dello 'storpio alla rovescia'» ${ }^{6}$. Corriamo il rischio di diventare degli esseri storpiati, solo orecchie. Questo è anche il tema de Il testimone auricolare ${ }^{7}$ di Elias Canetti, un insieme di racconti brevi che con humor riporta il brusìo dei pettegolezzi che compongono la trama di comunità chiuse.

Un ritratto d'infanzia 'auricolare' - Un testo di quaranta pagine, degli Archives du Patrimoine Autobiographique de la Belgique, racconta dell'esperienza infantile dell'autrice ricostruita sulla base dell'udito, portando a riflettere su quanto l'ascoltare costituisca una particolare forma di captazione percettiva della realtà. Muriel descrive l'incubo del sentire arrivare suo padre quasi sempre ubriaco, il chiudersi in camera con le sorelle, il cogliere tutti i passi che finivano col delineare la planimetria della casa, le urla, le violenze esercitate sulla madre: «Una sera quando le mie sorelle e io stessa eravamo a letto, sentivamo ancora che egli [il padre] rompeva tutto in casa. Sentivamo mia madre gridare e piangere, poi abbiamo inteso 't'ammazzo'. Mia madre ha cominciato a correre per casa, poi più niente. Sentivamo mio padre gridare 'non ritornare più', ecc., dopo è corso fuori e ha preso la sua automobile».

Akoustikoi - Se udire/intendere è una facoltà primordiale, proprio secondo il dettato di Freud «Il senso che il bambino sin prima della nascita non può chiudere», non sempre questa facoltà è stata esplorata a fondo, come il numero di parole in uso testimonia. In latino l'eco viene chiamata imago vocis, riconducendo così un fenomeno uditivo al visivo.

Il linguista Tullio de Mauro ha messo in evidenza quanto i termini con i quali si designa la voce (verba loquendi) siano molto più numerosi dei termini che designano l'udito e l'ascoltare:

\footnotetext{
${ }^{5}$ Jacques DERRIDA, Otobiographies, Paris, Ed. Galilée, 1984.

${ }^{6}$ Ivi, p. 107.

${ }^{7}$ Elias CANETTI, Il testimone auricolare, trad. di Gilberto FORTI e Raffaele ORIANI, Milano, Adelphi, 1995. Der Ohrenzeuge; Fünfzig Charaktere (München, 1974).

${ }^{8}$ Murielle FAGNANT, Ce livre va vous raconter une grosse partie d'une famille qui a vécu de terribles histoires, 2006, Dépôt APA BEL n 44, 42 pagine, p. 5. Il corsivo è mio.
} 
l'entendre: c'est dire que notre culture se préoccuperait davantage de définir le phénomène de la voix...

La relation entre l'ouïe (ou son manque) et l'expression verbale n'est pas attendue. Une sourde-aveugle dans le film extraordinaire de Herzog Le pays du silence et de l'obscurité dit: «on ne peut pas imaginer combien de bruits obsèdent une personne sourde».

Si on pense à l'importance accordée dans l'antiquité au silence, par les pythagoriciens par exemple (bien avant l'ordre des bénédictins), on comprend que la capacité d'être à l'écoute fut valorisée au maximum. Foucault le rappelle dans une conférence au Collège de France de 1982: les pythagoriciens demandaient aux disciples (akoustikoi) deux ans de silence, avant de pouvoir s'exprimer en devenant mathêmatikoi.

On raconte que Zénon le stoïcien dit à un jeune qui parlait trop: «Nous avons deux oreilles et une seule bouche, pour écouter plus et parler moins» (Diogène Laertius, Vitae philosophorum, VII 23).

Dialektikè, ou de l'art d'entrer en empathie - Edgar Radtke dans son intervention a rappelé que la parole dialecte - du latin dialectus, du grec dialektos, causer, [parole proche à dialektikè (téchné), art du discuter (dia=fra)] - signale proprement les conditions d'intimité que cette parole demande: un contexte d'empathie, de familiarité et de volonté de communication. Il a souligné que l'étude de la langue devrait prendre en charge ce caractère autobiographique du parler, ce caractère personnel étant plus marqué que dans la langue écrite. Et cela même si la langue écrite peut exprimer aussi divers aspects de l'oralité. On l'a vu durant le colloque à propos de l'écriture d'Assia Djebar, de Ferdinand Céline, et d'autres écrivains.

La recherche littéraire qui s'engage à reporter dans l'écriture la musicalité et la plurivocité linguistique est un travail de filigrane. 
in altre parole la nostra cultura si è preoccupata maggiormente di connotare il fenomeno voce ${ }^{9}$.

Definire la relazione arbitraria che collega la facoltà di esprimersi e l'universo dell'ascolto, non è semplice. Una sordo-cieca nello straordinario film di Herzog Il Paese del silenzio e dell'oscurită ${ }^{10}$ dice: «non si può immaginare quanto rumore assilli una persona che non sente».

Se pensiamo al rilievo dato nell'antichità al silenzio, dai pitagorici ad esempio (ben prima di ordini monastici come i benedettini), comprendiamo quanta importanza fosse data all'ascolto, all'accesso all'intendere. Lo ricorda Foucault in una conferenza al Collège de France del 1982: i pitagorici richiedevano ai discepoli (akoustikoi) due anni di silenzio prima di diventare mathêmatikoi con facoltà di parola ${ }^{11}$.

E si racconta che Zenone Stoico ad un giovane che parlava troppo disse: «abbiamo due orecchie e una sola bocca, per ascoltare di più e parlare di meno» (Diogene Laerzio, Vitae philosophorum, VII 23).

Dialektikè, o l'arte di entrare in empatia - Edgar Radtke durante il colloquio ha ricordato che la parola dialetto, - dal latino dialectus, dal greco dialektos, discorro, [parola prossima a dialektikè (téchné), arte del discutere (dia=fra)] - indica proprio quanto il dialetto stabilisca intimità, favorisca un contesto discorsivo di empatia, familiarità, volontà di comunicazione. Sottolinea quanto lo studio della lingua dovrebbe applicarsi di più a questo carattere autobiografico del parlato, carattere personale molto più marcato di quanto avvenga nella lingua scritta. Anche se la lingua scritta può esprimere molti aspetti dell'oralità. Lo abbiamo visto in una parte degli interventi relativi all'opera di Assia Djebar, e di Ferdinand Céline. La ricerca letteraria volta a riportare musicalità e plurivocalità linguistica nella scrittura è un fine lavoro di filigranatura.

\footnotetext{
${ }^{9}$ Tullio DE MAURO, «La voce, l'udito e lo spazio linguistico», in (a cura di) A. LEONI/F. CUTUGNO/M. PETTORINO/R.SAVY, Il parlato italiano, Atti del Convegno Nazionale di Napoli, 13 - 15 febbraio 2003, Napoli, D'Auria, 2004.

${ }^{10}$ Werner HERZOG, Land des Schweigens und der Dunkelheit, 1H25', 1971, film proiettato durante la rassegna Auto-bio-regia, che ha fatto seguito al colloquio.

11 Michel FOUCAULT, L’herméneutique du sujet, Cours au Collège de France (1981-1982), Paris, Gallimard/Seuil, 2001, pp. 396-397.
} 
De l'ouïe à la parole comme signe - La relation entre l'écoute et la parole est au centre de l'autobiographie d'Helen Keller (Alabama 1880 - 1968). Le film d'Arthur Penn (The Miracle Worker, 1957), tiré de cette autobiographie a été projeté pendant la session-cinéma qui a suivi le colloque.

The Story of my life nous raconte que le point de départ du difficile apprentissage de la parole (Helen était devenue sourde et aveugle à l'âge de 19 mois à la suite d'une congestion cérébrale) ainsi que la possibilité de sortir d'un monde sans contact sonore désormais avec l'extérieur ont été rendus possibles lorsqu'elle a pu évoquer le moment où elle avait prononcé toute petite le mot water, en établissant le premier rapport arbitraire entre un objet et sa dénotation. L'émergence dans sa mémoire d'un mot dit, prononcé, lui a permis petit à petit de reconstruire le monde de la langue, qui est par définition une convention.

La musique, l'original et ses répliques - Une anecdote fait état de ce que la mère en attente de Glenn Gould écoutait pendant des heures Bach. Glenn Gould a été le plus grand interprète des Variations Goldberg. Il abandonna la scène à trente-deux ans pour privilégier les auditoires d'enregistrement et la radio, anticipant sur une sensibilité qui sera reconnue dans la suite, en valorisant l'acte de la répétition et l'enregistrement plutôt que la musique life.

Gould chantonnait pendant qu'il jouait du piano et les techniciens du son, décidés à nettoyer les incises de sa voix, n'ont pas toujours atteint ce but. Gould préconisait de chanter involontairement, de suppléer en crescendo avec la voix aux impossibilités du piano, de réaliser la musique selon ce qu'il entendait dans sa tête. Gould se situe donc dans une contemporanéité absolue: celle de donner autant d'importance à l'original qu'à l'enregistrement des interprétations/variations.

Ah être seulement une voix! - C'est dans cette ligne qu'a travaillé Carmelo Bene, à partir d'un de ses premiers romans Credito italiano V.E.R.D.I. (1967). Tout au long de sa vie il a mis au centre de son œuvre l'ouïe et la répétition. 
Dall'ascolto all'arbitrarietà della parola - Il rapporto fra l'ascolto e la parola è al centro dell'autobiografia di Helen Keller (Alabama 1880 - 1968). Nella rassegna filmica Autobio-regia è stato proiettato il film di Arthur Penn (The Miracle Worker, 1957), tratto da questa autobiografia.

The Story of my life ci informa che il punto iniziale del difficile apprendimento della parola (Helen era diventata sorda e cieca all'età di 19 mesi a seguito di una congestione cerebrale), la sua possibilità di uscire da un mondo senza più contatti sonori con l'esterno, è stato l'evocare il momento di quando infante aveva pronunziato la parola water, acqua, stabilendo così il primo rapporto arbitrario, fra oggetto e denotazione. Questo emergere nella memoria del punto di contatto dell'ascolto con la parola, le ha consentito, con molto lavoro e fatica, di ricostruire la possibilità di parlare, di edificare il mondo di codici linguistici che sono per definizione convenzionali.

La musica: I'originale e le repliche - Un aneddoto racconta che la mamma in attesa di Glenn Gould ascoltava per ore Bach. Glenn Gould è stato il più grande interprete del '900 delle Variazioni Goldberg. Abbandonò le scene a 32 anni, privilegiando le sale di registrazione e la radio, - anticipatore per questo di una sensibilità che in seguito avrà un percorso riconosciuto -, valorizzando cosí l'atto del ripetere, registrare, piuttosto che di esaltare la musica life.

Gould canticchiava mentre suonava, e i tecnici del suono, impegnati a pulire le incisioni dalla sua voce non sono sempre riusciti allo scopo. Gould diceva di cantare involontariamente, di sopperire in crescendo con la voce alle impossibilità del pianoforte, di realizzare la musica secondo i suoi intendimenti, secondo ciò che sentiva nella testa ${ }^{12}$.

Gould si posiziona in una contemporaneità assoluta: quella di dare importanza tanto all’originale quanto alla registrazione delle varie interpretazioni/variazioni.

Ah essere solo una voce! - È proprio su questa linea che si è mosso Carmelo Bene: lo scambio fra gli originali e le repliche é stato l'essenza del suo lavoro. Sin dal romanzo Credito italiano V.E.R.D.I. (1967), l'udito appare il canale principale di comunicazione col mondo, per divenire poi il punto focale di tutto il suo teatro.

\footnotetext{
${ }^{12}$ Glenn GOULD, «Le 'variazioni Goldberg'», in L'ala del turbine intelligente, trad. dall'inglese di Anna BASSAN LEVI, Milano, Adelphi, 1988, p. 63. The Glenn Gould reader, 1984.
} 
De Carmelo Bene nous avons écouté pendant le colloque un inédit audio ' $L$ mal de' fiori (8', 2000). Luisa Viglietti et Francesca Oppedisano ont mis l'accent sur la synergie de ses réflexions théoriques et de son être sur les tréteaux.

CB a dégagé la parole de toute illusion référentielle. Ah essere solo una voce!, Ah être seulement une voix!

L'utilisation du play back, la dissociation de la voix du gestuel, marque en CB la valeur impérative de la langue (qui nous précède), la langue marionnettiste, par rapport à notre parole, en estompant aussi l'illusion de pouvoir utiliser le gestuel pour accentuer la parole.

Privilégier le domaine du silence, le manque lacanien, faire obstacle à, entraver l'obvious du déjà dit, tout cela rythme les remakes d'œuvres de Shakespeare, de Laforgue, de Von Kleist, de De Sade (auteurs dont les œuvres ont été passées au tamis par CB), dans lesquels nous sommes poussés à expulser le mort oral (le re-citer), à habiter cet hiatus, cet espace entre langue et parole, entre l'univers des sons et l'utilisation active de l'expression (ton et grain de la voix). Exactement comme rappelle CB dans son ouvrage L'orecchio mancante, en mentionnant Epictète: «La sagesse est le chuchotement du solitaire à soi-même en plein marché».

Toute son œuvre livre une réflexion sur l'ouïr, l'entendre, le méprendre:

«C’est une prémisse fondamentale reconnaître immédiatement que le son amplifié, c'est le revers d'une prothèse de facilité. Prothèse est pour le dire brièvement une rallonge de la portée orale, utilisée dans le seul but de consentir à qui prêche d'être ouï d'une multitude excédant la capacité usuelle de salles et places [...] Je ne peux pas ne pas rappeler que [...] l'étymon qualifie l'acteur acte-rhétorique de l'agir oral: agere (tout autre que bouger frénétiquement dans l’espace scénique).

[...] Aussi dans l'animal humain la perception du son précède - et de combien! - sa naissance: ce venir dans l'obscurité (rien à voir avec la lumière). Dans les eaux maternelles nous nous écoutons: nous sommes informés des bruits (électro)domestiques extérieurs. Nous enregistrons passivement, grumeaux d'un discours qui ne nous appartient pas (qui dans la vie à suivre - j'ai dit ailleurs - n'appartiendra plus au sujet parlant). Quant à nous-voir, une fois au 'monde', nous devrons patienter pas mal de jours. Dans notre aventure physiologique, c'est donc à l'audio de précéder le visuel. En dépit de la rapidité de la lumière». 
Abbiamo ascoltato durante l'incontro un inedito audio di Carmelo Bene ' $L$ mal de' fiori (8', 2000). Luisa Viglietti e Francesca Oppedisano hanno posto l'accento su quanto importante in CB sia stata la sinergia fra la riflessione teorica e il suo prodursi in scena.

$\mathrm{CB}$ ha svincolato la parola da ogni illusione contenutistica. Ah essere solo una voce!

L'uso del play back, il dissociare la voce dal gesto, marca in CB il valore imperativo della langue (che ci precede), la langue burattinaio, rispetto alla nostra parole, e rispetto all'illusione di poter usare il gesto per avvalorare, enfatizzare la parole.

In CB il dominio del silenzio, il manque lacaniano, l'inceppare l'ovvietà del già detto, fa esplodere il patrimonio acclarato di Shakespeare, di Laforgue, di Von Kleist, di De Sade (autori le cui opere sono state passate al setaccio da CB). Siamo letteralmente spinti ad espellere il morto orale (il re-citare), ad abitare lo iato, esplorandone lo spazio e il vuoto, fra langue e parole, fra l'universo dei suoni e l'uso attivo dell'espressione (tono e grain de la voix) per divenire responsabili di intendere, di ascoltare. Proprio come ricorda CB, citando Epitteto, nel suo libro L'orecchio mancante ${ }^{13}$ : «La saggezza è il bisbiglio del solitario a se stesso in pieno mercato».

Tutta la sua opera conduce ad una riflessione sull'udire e l'intendere, il fraintendere:

«È premessa fondamentale riconoscere subito che il suono amplificato è il rovescio d'una protesi di comodo. Protesi è, a dirla in breve, una 'prolunga' della portata orale, utilizzata al solo scopo di consentire a chi predica d'essere udito da una moltitudine eccedente la capienza usuale di sale e piazze [...] Né posso non ricordare che [...] l'etimo qualifichi l'attore attoretorico dell'agire orale: agere (altro che sfaccendare nello spazio scenico).

[...] Anche nell'animale umano la percezione del suono precede - ma di tanto! - la sua nascita: questo venire al buio (altro che luce). Nelle acque materne ci-sentiamo: siamo informati dai rumori (elettro)domestici esterni. Registriamo passivamente i grumi di un discorso che non ci appartiene (che nella vita a seguire - ho detto altrove - non apparterrà più al soggetto parlante). Quanto a veder-ci, una volta al 'mondo' dovremo pazientare un bel po' di giorni. Nella nostra avventura fisiologica è dunque l'audio a precedere il visivo. A dispetto della velocità del suono e della luce» ${ }^{14}$.

\footnotetext{
${ }^{13}$ Carmelo BENE, L'orecchio mancante, Milano, Feltrinelli, 1970.

${ }^{14}$ C. BENE, «Autografia d'un ritratto», Opere, Milano, Bompiani, 2002, pp. XIV-XV. Il suono amplificato non è da intendersi dunque, come una protesi tecnologica, ma come résonnance.
} 